\title{
Construction of a mariner-based transposon vector for use in insertion sequence mutagenesis in selected members of the Rhizobiaceae
}

\author{
Benjamin J Perry and Christopher K Yost
}

\begin{abstract}
Background: The Rhizobiaceae family of Gram-negative bacteria often engage in symbiosis with plants of economic importance. Historically, genetic studies to identify the function of individual genes, and characterize the biology of these bacteria have relied on the use of classical transposon mutagenesis. To increase the rate of scientific discovery in the Rhizobiaceae there is a need to adapt high-throughput genetic screens like insertion sequencing for use in this family of bacteria. Here we describe a Rhizobiaceae compatible Mmel-adapted mariner transposon that can be used with insertion sequencing for high-throughput genetic screening.

Results: The newly constructed mariner transposon PSAM_RI mutagenized R. leguminosarum, S. meliloti, and A. tumefaciens at a high frequency. In $R$. leguminosarum, mutant pools were generated that saturated $88 \%$ of potential mariner insertions sites in the genome. Analysis of the $R$. leguminosarum transposon insertion sequencing data with a previously described hidden Markov model-based method resulted in assignment of the contribution of all annotated genes in the $R$. leguminosarum 3841 genome for growth on a complex medium. Good concordance was observed between genes observed to be required for growth on the complex medium, and previous studies.

Conclusions: The newly described Rhizobiaceaee compatible mariner transposon insertion sequencing vector pSAM_Rl has been shown to mutagenize at a high frequency and to be an effective tool for use in high-throughput genetic screening. The construction and validation of this transposon insertion sequencing tool for use in the Rhizobiziaceae will provide an opportunity for researchers in the Rhizobiaceae community to use high-throughput genetic screening, allowing for significant increase in the rate of genetic discovery, particularly given the recent release of genome sequences from many Rhizobiaceae strains.
\end{abstract}

\section{Background}

Insertion sequencing (INSeq) is a technique for high throughput forward genetic screening that has recently become a favorable approach to studying gene function at the genome scale $[1,2]$. INSeq relies on the use of next-generation DNA sequencing to audit the presence of hundreds of thousands of unique transposon insertions present in a pool of mutants that collectively saturate that organism's genome with transposition events [3-6]. In general, INSeq based methods can use two different methods to analyze gene function. The first relies on sequencing the transposon insertions sites in an

\footnotetext{
*Correspondence: chris.yost@uregina.ca
Department of Biology, University of Regina, 3737 Wascana Parkway, Regina,

*Correspondence: chris.yost@uregina.ca
Department of Biology, University of Regina, 3737 Wascana Parkway, Regina, SK S4S OA2, Canada
}

input pool and an output pool of transposon mutants, and using the differential representation of mutants in each pool to infer the functional role of each gene with sufficient representation of insertion sites [7]. The second method relies on creating a mutant pool sufficiently large and complex that it saturates the genome and allows for analysis of regions with statistically fewer, or no, insertions than expected using a non-parametric [8], Bayesian model [9], or hidden Markov model (HMM) based analysis [10,11]. Both approaches have been applied to several species of bacteria to investigate genes involved in colonization of hosts [12-14], resistance to antibiotics [15], characterizing metabolic pathways [16,17], deducing core essential genomes [18-24], and recently, examining genes involved in colonizing soil environments [7]. 
The Rhizobiaceae is a family of alpha-proteobacteria containing three agriculturally important genera of soil bacteria: Rhizobium, Sinorhizobium and Agrobacterium [25]. Members in these genera share a unique relationship with plant hosts. Rhizobium, and Sinorhizobium are both able to enter into an endosymbiotic mutualism with certain species of leguminous plants, in which the Rhizobia fix atmospheric nitrogen into a biologically available form for the plant in return for fixed carbon and energy [26]. This symbiosis is particularly important in the context of agriculturally produced pulse crops, where the Rhizobium legume symbiosis affords farmers the ability to reduce the rate of synthetic nitrogen fertilizers application [27]. Conversely, the relationship of Agrobacterium with its plant host is parasitic. In this symbiosis, Agrobacterium infects the tissues of a plant host and transforms specific virulence genes into the host's DNA, resulting in tumorgenic growth with altered cellular metabolism that the bacteria then colonize [28]. The formation of several galls at the stem root interface results in a plant infection known as crown gall, that can have a significant impact on the crop yield of stone fruits, berries, and nuts [29].

Genetic research in Rhizobium, Sinorhizobium, and Agrobacterium has relied heavily on the use of transposon mutagenesis screens. Perhaps the most commonly used transposon in the Rhizobiaceae is the Tn5 transposon [30-32]. The use of Tn5 genetic screens is numerous and has helped to elucidate genes involved in metabolism [33-35], desiccation tolerance [36,37], and cell envelope physiology [38] for example. Implementation of transposon mutagenesis with the high-throughput techniques of INSeq promise to accelerate the rate at which genetic research in the Rhizobiaceae is currently performed. Furthermore, it would allow for comprehensive genome screens for genes involved in host interactions, metabolism, survival, and possibly plasmid maintenance, under any testable condition.

The mariner class of transposon is a host independent transposon that unlike the random insertion transposons such as $\operatorname{Tn} 5$ is known to specifically insert into an organism's genome at thymine-adenine ('TA') motifs [39]. Because of this defined insertion preference, transposition events can be modeled in silico in any sequenced genome to understand the defined number of insertion locations that exist. This type of analysis can be further refined to examine insertions per gene or within any defined region of interest in the genome. Furthermore, using a transposon with a defined number of insertion loci allows for robust statistical analysis when used in a transposon insertion sequencing methodology. One such analysis package uses a HMM to predict the essentiality of every gene under a particular growth condition in an organism's genome given a sufficiently dense INSeq data set [10]. The advantages of this type of analysis is that it does not solely rely on the comparison of an input and output pool of mutants as it uses statistical inference on a defined number of insertion sites to infer an over represented or under represented number of insertions within a given region.

Here we describe the modification of a previous INSeq mutagenesis vector pSAM_Bt, which uses a mariner class transposon with modified IR elements, to selectively capture $15-16 \mathrm{bp}$ of genomic DNA adjacent to the transposon insertion [4]. The DNA fragment is then processed for next-generation DNA sequencing. The new INSeq vector uses a Rhizobiaceae specific promoter to drive the expression of the plasmid-borne transposase, and has had the erythromycin resistance gene cassette replaced with a neomycin resistance gene cassette (ntpII) for ease of selection in the Rhizobiaceae. The host range of transposition with pSAM_Rl was examined and an INSeq experiment was performed on the model organism Rhizobium leguminosarum bv. viciae 3841 (RLV3841) to examine the insertion density that the PSAM_Rl transposon could achieve. Furthermore, the functionality of the transposon in RLV3841 for INSeq was also examined.

\section{Results}

\section{Construction of pSAM_RI and transposition frequency} within the Rhizobiaceae

The MmeI-adapted mariner transposon suicide delivery vector PSAM_Rl was constructed from a previously described MmeI-adapted mariner delivery vector PSAM_Bt [4]. To do so, the 974 bp Ery ${ }^{\mathrm{R}}$ in pSAM_Bt was replaced with a $979 \mathrm{bp} \mathrm{Neo/Kan}{ }^{\mathrm{R}}$ resistance cassette from pSC189 and the 304 bp Bacteroides thetaiotaomicron rpoD promoter region was replaced with a 366 bp RLV3841 rpoD promoter region. A map of the pSAM_Rl construct is shown in Figure 1, with restriction enzyme sites used for cloning indicated. The pSAM_Rl construct was maintained in E. coli strain SM10גpir as this strain harbours both the $\lambda$ pir gene, required for the replication of R6Ky oriR, and chromosomally integrated tra genes, required for conjugative transfer via the plasmid borne RP4-oriT. Analysis of the transposition frequency of the pSAM_Rl mariner transposon was evaluated via conjugative transfer of the pSAM_Rl suicide vector from E. coli SM10גpir into R. leguminosarum, S. meliloti, and A. tumefaciens. Transposition frequency was highest in RLV3841, yielding an average of $2.01 \times 10^{-4}$ transposon mutants per recipient cell. The frequencies of transposition in A. tumefaciens and $S$. meliloti were observed to be $8.04 \times 10^{-5}$ and $2.54 \times 10^{-5}$ mutants per recipient cells, respectively.

\section{Analysis of PSAM_RI transposition in the RLV3841 genome} Analysis of PSAM_Rl transposon integration was performed using three independent pools of $\sim 1.9 \times 10^{6}$ transposon mutants. Mutant pools were grown for approximately 16 


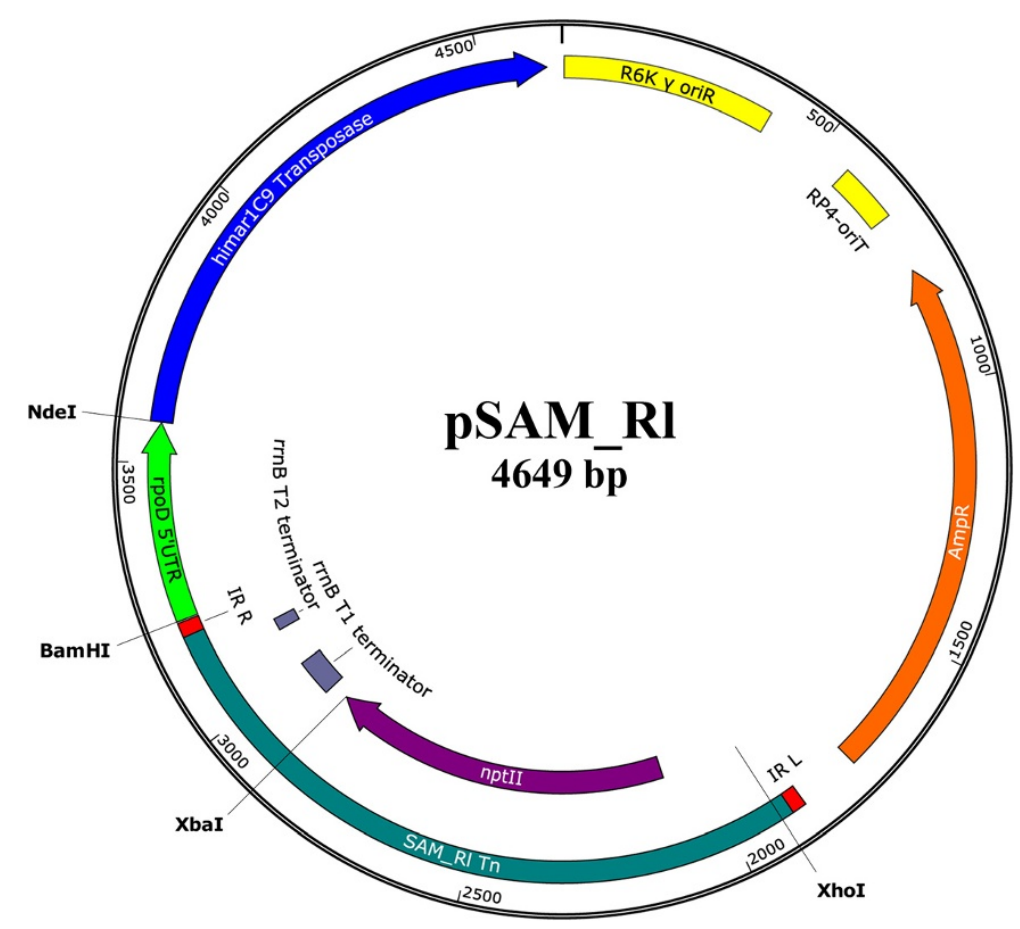

Figure 1 pSAM_RI plasmid map. Restriction enzymes used for cloning are indicated. Antibiotic markers (ampicillin, AmpR; neomycin/kanamycin, nptl), origin of replication (R6K y oriR), origin of transfer (RP4-oriT), transposase (himar1C9), tranposase promoter (rpoD 5'UTR), Mmel-adapted mariner inverse repeats ( $\left.I R \_R, I R \_L\right)$, transposon borne Rho-independant terminator ( $r m B$ T1, rrnB T2). Plasmid map produced using Snapgene software.

generations (48 hours) on TY basal medium. The mutants were collected en masse and the 15-16 bp of genomic DNA adjacent to the transposon insertion was sequenced using a modified INSeq method on the Ion Torrent PGM. Transposon insertion sequences from the three replicate samples were then pooled and aligned onto the RLV3841 genome, resulting in 2,319,239 usable transposon insertion tags (Tn-tags) for analysis with the transposon HMM (Tn-HMM) python module [10]. From in silico analysis it was calculated that 140,056 potential mariner transposon insertion sites existed within the
RLV3841 genome. Of these, 116,544 unique transposon insertions were observed in the composite data collected from the three PSAM_Rl mutant pools grown on TY agar. This corresponded to an overall insertion density of $83 \%$ in the RLV3841 genome, with insertion density across replicons ranging between $68 \%$ to $88 \%$ (Table 1). The mean read count per mariner insertion site, observed to have a transposon insert, ranged from 18.57 to 22.09 sequence reads per insertion across all replicons with the median read count per insertion ranging from 10.0 to 13.0 reads per insertion (Table 1).

Table 1 Summary of pSAM_RI transposition in RLV3841

\begin{tabular}{|c|c|c|c|c|c|c|c|c|}
\hline Replicon & Size (bp) & GC\% & Gene number & Potential insertions & Observed insertions & Insertion density ${ }^{A}$ & Mean read count ${ }^{B}$ & $\begin{array}{l}\text { Median read } \\
\text { count }^{\mathrm{C}}\end{array}$ \\
\hline Chrom & 5057142 & 61.10 & 4800 & 89660 & 73375 & 0.82 & 20.46 & 13.00 \\
\hline RL12 & 870021 & 61.00 & 790 & 14845 & 13122 & 0.88 & 18.57 & 12.00 \\
\hline RL11 & 684202 & 61.00 & 644 & 12456 & 10964 & 0.88 & 19.97 & 13.00 \\
\hline RL10 & 488135 & 59.60 & 471 & 10003 & 8470 & 0.85 & 17.19 & 10.00 \\
\hline RL9 & 352782 & 61.00 & 313 & 6359 & 5453 & 0.86 & 19.65 & 13.00 \\
\hline RL8 & 147463 & 58.70 & 142 & 3118 & 2702 & 0.87 & 17.96 & 11.00 \\
\hline RL7 & 151546 & 57.60 & 186 & 3615 & 2458 & 0.68 & 22.09 & 12.00 \\
\hline Genome & 7751291 & 60.00 & 7346 & 140056 & 116544 & 0.83 & 19.41 & 12.00 \\
\hline
\end{tabular}

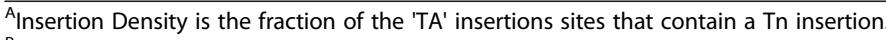

${ }^{B}$ Mean Read Count is the mean number of Tn insertions within 'TA' sites.

$\mathrm{C}^{\mathrm{C}}$ Median Read Count is the median number of $\mathrm{Tn}$ insertions within a specific 'TA' site. 


\section{Distribution of phenotypic classes throughout the RLV3841 genome}

Each RLV3841 replicon was analyzed using the Tn-HMM python module [10] to classify genes based on the observed density of $\mathrm{Tn}$ insertions in each gene within the mixed mutant cell population. For example, a gene which had no detectable Tn insertion sites following DNA sequencing from the pooled mutant DNA suggests cells carrying a mutation in this gene were not maintained in the population. These genes are described as conferring an essential phenotype. The procedure is described in greater detail in the Materials and Methods.

The seven replicons of RLV3841 were analyzed separately to account for variations in mean read depth, insertion density, and median read count between the replicons that may have been a result of their independent replication within the cell. Across all replicons 89.5\% of the genes were observed to have a neutral or negligible impact on the ability of RLV3841 to grow on TY medium when disrupted by the transposon (Table 2). Genes identified as conferring a phenotype classification of essential, growth-defective, and growth-advantage were observed to represent $5.6 \%, 4.0 \%$, and $0.1 \%$ of the genes within the genome, respectively. Throughout the genome, 10 genes contained no 'TA' transposon insertions sites and as such could not be assigned to a growth effect state. However this represents only $0.14 \%$ of the total predicted genes within the genome (Table 2).

\section{Specific essential genes within the RLV3841 genome}

The defined phenotypic class of several house-keeping genes involved in ATP synthesis, cell divisions, DNA replication, and RNA transcription are summarized in Table 3. Additionally, Table 3 summarizes the essential nature of plasmid specific replication genes for each of

Table 2 Summary of phenotypic classes across the RLV3841 genome

\begin{tabular}{lllllll}
\hline Replicon & $\begin{array}{l}\text { Mapped } \\
\text { reads }\end{array}$ & \multicolumn{6}{c}{ Genes per specific phenotypic class } \\
\cline { 3 - 7 } & ES & GD & NE & GA & No Data $^{\text {B }}$ \\
\hline Chrom & 1501032 & 317 & 272 & 4176 & 35 & 0 \\
pRL12 & 243699 & 8 & 3 & 774 & 3 & 2 \\
pRL11 & 218944 & 15 & 9 & 614 & 3 & 3 \\
pRL10 & 145621 & 15 & 3 & 447 & 3 & 3 \\
pRL09 & 107144 & 7 & 1 & 305 & 0 & 0 \\
pRL08 & 48512 & 3 & 1 & 137 & 1 & 0 \\
pRL07 & 54287 & 44 & 8 & 124 & 8 & 2 \\
\hline
\end{tabular}

$\mathrm{ES}=$ Essential, $\mathrm{GD}=$ Growth-defective, $\mathrm{NE}=$ Neutral, GA = Growth-advantage ${ }^{A}$ Genes were designated to a phenotypic class based on the state most frequently observed in the TA sites within the boundaries of an annotated gene, except in the case of essential genes which could also have been assigned to the ES state if they contained a stretch of ES TA sites that was statistically significant based on the extreme value distribution. Please refer to the Materials and Methods section for more detail.

${ }^{B}$ Genes lacking TA motifs. the 6 plasmids contained in the RLV3841 genome that are required for plasmid maintenance during cell division. From previous analysis [38], genes known to be required for growth on TY medium are summarized at the end of Table 3 with their essential nature, as determined by the 4 phenotypic classes, reported by the Tn-HMM software.

\section{Discussion}

High throughput forward genetic screening is rapidly being adopted in a diverse range of organisms. INSeq, and similar high-throughput techniques, have been used in several bacterial species, including Salmonella [5], Mycobacterium [17], Haemophilus [6], Vibrio [40], Pseudomonas [15], Chronobacteria [24] and Bacillus [7]. The technique is very attractive as it allows for the high-throughput functional screening of an organism's genome under varied growth conditions. Here we present the design and validation of a transposon mutagenesis system that will allow for the application of high-throughput INSeq genetic screening for use within the Rhizobiaceae.

\section{Transposon mutagenesis with pSAM_RI in RLV3841}

We have successfully demonstrated the implementation of a mariner class transposon to mutagenize selected species within the family Rhizobiaceae. We found that the MmeI-adapted mariner transposon harbored on pSAM_Rl could mutagenize R. leguminosarum, A. tumefaciens, and S. meliloti at a high frequency. In the case of RLV3841, it was observed that this high frequency of mutagenesis was dense enough to generate saturating libraries of transposon insertion mutants. We were able to generate transposon mutant libraries that saturated the RLV3841 genome with an insertion density of 0.88 , which is higher then the insertion density of the data set used for validation of the HMM used for analysis [10]. This suggests that the combination of mariner based transposon insertion sequencing with the Bayesian based HMM analysis would yield accurate and full genome level results. Furthermore, our analysis used approximately 2.3 M reads. And increased read depth could help increase confidence in the analysis, particularly in resolving the phenotypic classifications of essential and growth defective.

We also observed no bias of mariner transposon insertion across the RLV3841 chromosome and plasmids, suggesting that the transposon inserts randomly and therefore should allow for reliable whole-genome screening approaches. Analysis of read depth showed that there was also little bias in mean read-count per insertion, except in the case of pRL7, which was slightly higher than the other replicons. We suggest that this is a result of pRL7 being maintained at a higher copy number than the other megaplasmids and chromosome, and 
Table 3 Selected genes in RLV3841 cultured on TY agar with Tn insertions substantially below expected levels

\begin{tabular}{|c|c|c|c|c|c|c|}
\hline Locus & Gene & Gene annotation & $\begin{array}{l}\text { Potential } \\
\text { insertions }{ }^{\mathrm{A}}\end{array}$ & $\begin{array}{l}\text { Insertion } \\
\text { density }^{\mathrm{B}}\end{array}$ & $\begin{array}{l}\text { Mean read } \\
\text { count }^{c}\end{array}$ & $\begin{array}{l}\text { Phenotypic } \\
\text { class }{ }^{\mathrm{D}}\end{array}$ \\
\hline \multicolumn{7}{|c|}{ Selected chromosomal house keeping genes } \\
\hline RL0924 & atpl & Putative ATP synthase I & 4 & 0.000 & 0.000 & ES \\
\hline RL0925 & $a t p B$ & FOF1 ATP synthase subunit A & 12 & 0.083 & 1.000 & ES \\
\hline RL0926 & $\operatorname{atpC}$ & FOF1 ATP synthase subunit $C$ & 7 & 0.000 & 0.000 & ES \\
\hline RL0927 & $\operatorname{atpG}$ & FOF1 ATP synthase subunit B' & 7 & 0.000 & 0.000 & ES \\
\hline RL0928 & $a t p F$ & F0F1 ATP synthase subunit B & 4 & 0.000 & 0.000 & ES \\
\hline RL4405 & $\operatorname{atpC}$ & FOF1 ATP synthase subunit Epsilon & 3 & 0.333 & 1.000 & ES \\
\hline RL4407 & $a t p D$ & F0F1 ATP synthase subunit Beta & 23 & 0.043 & 1.000 & ES \\
\hline RL4408 & $\operatorname{atpG}$ & FOF1 ATP synthase subunit Gamma & 12 & 0.083 & 1.000 & ES \\
\hline RL4409 & $\operatorname{atpA}$ & F0F1 ATP synthase subunit Alpha & 22 & 0.000 & 0.000 & ES \\
\hline RL4410 & atpH & FOF1 ATP synthase subunit Delta & 8 & 0.125 & 1.000 & ES \\
\hline RL4412 & priA & Primosome assembly protein PriA & 23 & 0.261 & 4.000 & GD \\
\hline RL3408 & $d n a G$ & DNA primase & 23 & 0.000 & 0.000 & ES \\
\hline RL3298 & $f t s Z$ & Cell division protein FtsZ & 9 & 0.000 & 0.000 & ES \\
\hline RL3299 & $\mathrm{fts} A$ & Putative cell division protein FtsA & 12 & 0.083 & 1.000 & ES \\
\hline RL3300 & $f t s Q$ & Putative cell division protein FtsQ & 14 & 0.000 & 0.000 & ES \\
\hline RL3308 & $f t s W$ & Putative cell division protein FtsW & 13 & 0.000 & 1.000 & ES \\
\hline RL3965 & $\mathrm{ftsH}$ & Putative cell division protein FtsH & 19 & 0.105 & 1.500 & ES \\
\hline RL2515 & gyrB & DNA topoisomerase IV subunit B & 26 & 0.115 & 9.333 & ES \\
\hline RL2401 & gyrA & DNA gyrase subunit A & 34 & 0.059 & 1.000 & ES \\
\hline RL1723 & $d n a E$ & DNA polymerase III subunit Alpha & 51 & 0.078 & 1.000 & ES \\
\hline RL4697 & $d n a E$ & Putative DNA polymerase III subunit Alpha & 76 & 0.961 & 23.562 & $\mathrm{NE}$ \\
\hline RL0334 & $d n a N$ & DNA polymerase III subunit Beta & 14 & 0.071 & 1.000 & ES \\
\hline RL2099 & red & Putative single-stranded-DNA-specific exonuclease & 17 & 0.294 & 3.800 & $\mathrm{GD}$ \\
\hline RL1766 & rрoB & DNA-directed RNA polymerase subunit Beta & 64 & 0.016 & 1.000 & ES \\
\hline RL1767 & rpoC & Putative DNA-directed RNA polymerase subunit Beta' & 61 & 0.066 & 1.750 & ES \\
\hline RL1798 & rpoA & Putative DNA-directed RNA polymerase subunit Alpha & 12 & 0.000 & 0.000 & ES \\
\hline RL0059 & - & Putative ATP-dependant helicase & 18 & 0.778 & 21.714 & $\mathrm{NE}$ \\
\hline RL0582 & - & Putative ATP-dependant helicase & 39 & 0.949 & 18.730 & $\mathrm{NE}$ \\
\hline RL1551 & $d n a C$ & Putative replicative DNA helicase & 24 & 0.042 & 24.000 & ES \\
\hline \multicolumn{7}{|c|}{ Selected plasmid borne genes } \\
\hline pRL120001 & repA & Putative replication protein $\mathrm{A}$ & 21 & 0.000 & 0.000 & ES \\
\hline pRL120002 & repB & Putative replication protein B & 9 & 0.000 & 0.000 & ES \\
\hline pRL120003 & repC & Putative replication protein $C$ & 13 & 0.000 & 0.000 & ES \\
\hline pRL110001 & repA & Putative replication protein $\mathrm{A}$ & 25 & 0.040 & 1.000 & ES \\
\hline pRL110002 & repB & Putative replication protein B & 16 & 0.063 & 1.000 & ES \\
\hline pRL110003 & repC & Putative replication protein $C$ & 15 & 0.067 & 1.000 & ES \\
\hline pRL100001 & repA & Putative RepA replication protein & 19 & 0.000 & 0.000 & ES \\
\hline pRL100002 & repB & Putative RepB replication protein & 6 & 0.000 & 0.000 & ES \\
\hline pRL100003 & repC & Putative RepC replication protein & 22 & 0.045 & 1.000 & ES \\
\hline pRL90001 & repA & Putative replication partitioning protein & 30 & 0.000 & 0.000 & ES \\
\hline pRL90002 & rep $B$ & Putative replication partitioning protein & 19 & 0.053 & 1.000 & ES \\
\hline pRL90003 & repC & Putative replication initiation protein RepC & 15 & 0.000 & 0.000 & ES \\
\hline
\end{tabular}


Table 3 Selected genes in RLV3841 cultured on TY agar with Tn insertions substantially below expected levels (Continued)

\begin{tabular}{|c|c|c|c|c|c|c|}
\hline pRL80001 & repA & Putative replication protein RepA & 38 & 0.000 & 0.000 & ES \\
\hline pRL80002 & rep $B$ & Putative replication protein RepB & 23 & 0.043 & 1.000 & ES \\
\hline pRL80003 & repC & Putative replication initiation protein $\mathrm{RepC}$ & 28 & 0.036 & 1.000 & ES \\
\hline pRL70092 & repA & Putative replication protein & 34 & 0.529 & 1.722 & $\mathrm{GD}$ \\
\hline pRL70093 & rep $B$ & Putative replication protein $B$ & 22 & 0.455 & 1.100 & $\mathrm{GD}$ \\
\hline pRL70094 & repC & Putative replication initiation protein RepC & 33 & 0.455 & 1.200 & GD \\
\hline \multicolumn{7}{|c|}{ Previously experimentally confirmed genes with a TY- defective phenotype } \\
\hline RL4692 & $\operatorname{ctp} A$ & Putative carboxy-terminal processing protease precursor & 14 & 0.429 & 5.500 & $\mathrm{GD}$ \\
\hline RL3501 & - & Conserved hypothetical membrane protein & 42 & 0.667 & 3.107 & $\mathrm{GD}$ \\
\hline RL2815 & fabF2 & 3-Oxoacyl acyl carrier protein synthase & 11 & 0.364 & 1.000 & GD \\
\hline RL1375 & phaD2 & Putative $\mathrm{Na}+/ \mathrm{H}+$ antiporter subunit $\mathrm{D}$ & 26 & 0.192 & 1.400 & $\mathrm{GD}$ \\
\hline \multicolumn{7}{|c|}{ Examples of genes with higher than expected $T n$ insertions ${ }^{\mathrm{E}}$} \\
\hline RL0868 & - & Putative lipid A oxidase & 16 & 1.000 & 113.688 & GA \\
\hline RL2661 & - & Putative transmembrane component of $\mathrm{ABC}$ transporter & 13 & 1.000 & 62.846 & GA \\
\hline RL0684 & - & Putative transmembrane protein & 27 & 0.963 & 64.346 & GA \\
\hline
\end{tabular}

$\mathrm{ES}=$ Essential, GD = Growth Defect, $\mathrm{NE}=$ Neutral, GA = Growth Advantage.

${ }^{A}$ Potential insertions is the number of 'TA' nucleotide motifs within the gene.

${ }^{B}$ Insertion density is the fraction of all 'TA' insertions sites with a Tn insertion.

${ }^{C}$ Mean read count is the mean number of Tn insertions at 'TA' sites with a Tn insertion.

${ }^{D}$ Genes were designated to a phenotypic class based on the state most frequently observed in the TA sites within the boundaries of an annotated gene, except in the case of essential genes which could also have been assigned to the ES state if they contained a stretch of ES TA sites that was statistically significant based on the extreme value distribution. Please refer to the Materials and Methods section for more detail.

EThree genes with the phenotypic classification of Growth-advantage were included to provide context to the insertional densities and mean read count of the Growth-advantage state compared to the essential state classification.

so sampling of the mutant pool DNA would results in a higher sampling of pRL7 transposon insertion tags than the other plasmids, or chromosome. When the increase in mean read counts is taken into consideration with the insertion density, it appears that although the higher copy number of pRL7 resulted in a higher mean read depth, it did not increase the insertions density, suggesting that the saturation of pRL7 had reached a plateau and an increased presence of pRL7 did not result in a corresponding increase in the number of unique insertions sites.

Analysis of RLV3841 INSeq using a hidden Markov model Analysis of the TY INSeq data set with the Tn-HMM analysis package assigned accurate phenotypic classification to several genes thought to be essential housekeeping genes necessary for growth under normal conditions. The analysis showed that insertions in genes required for ATP synthesis were absent in the mutant pools. These genes are expected to be essential due to their central role in metabolism and their designation as essential in this assay supports the validity of using the MmeI-adapted mariner in conjunction with the HMM analysis as our INSeq methodology. Furthermore, visual investigation of the transposon insertion density around the region encoding the ATP synthase genes revealed a high insertion density leading up to and after the genes, further supporting the conclusion that the technique is robust and can discern regions of essentiality from those of other states at a high resolution.

In a few instances, a gene expected to be essential was observed to be neutral. RL4697 is annotated as a putative DNA polymerase III alpha sub-unit, and is therefore predicted to be required for proper DNA polymerase function; however, the gene was classified in the neutral category with $96.1 \%$ of all potential insertion sites in the locus observed to have insertions. We suggest that RL4697 may be misanotated, as RL1723, another DNA polymerase III alpha subunit, was observed to be essential (Table 3). This highlights another potential use of INSeq in the Rhizobiaceae for validation and quality improvement of genome annotations.

Five of the megaplasmids in the RLV3841 genome were observed to have a set of 3 plasmid replication genes that the INSeq approach identified as essential for plasmid replication and maintenance. The exception was pRL7 which has two sets of replication genes [41] and therefore functional redundancy may have complicated the the classification of the pRL7 rep genes into the phenotypic classes. The classification of the rep genes on each plasmid as essential provides validation of the INSeq method. Tn insertion within a replication locus would result in the loss of the plasmid from the mutant cell populations harvested for DNA extraction and 
INSeq DNA sequencing. This result highlights the value of the INSeq approach in the genetic characterization of novel plasmids, as the method is able to identify plasmid encoded genes that are required for plasmid replication and maintenance. Furthermore the method can identify plasmid-encoded genes that provide a fitness advantage to the host under specific growth conditions based on an observed low Tn insertion density.

The genes from previously described mutants with growth defects on TY medium were also examined. Previous work identified [38] several genes that are important for growth on TY medium. When we compared those results with the results produced by the INSeq analysis of RLV3841 grown on TY medium we observed good concordance with these previously published results. In our results, the four TY related genes were all observed to result in a growth-defective phenotype after $\sim 16$ generations of growth, which is in agreement that the interruption of these genes via mutagenesis will result in impaired growth on TY.

\section{Conclusions}

The construction and validation of the mariner pSAM_Rl transposon delivery vector as a transposon insertion sequencing tool for use in the Rhizobiziaceae will provide an opportunity for researchers in the Rhizobiaceae community to use a new high throughput genetic screening approach. There are many research opportunities within the Rhizobiaceae that could be examined using the INSeq methodology. For example, use of a INSeq approach in rhizobia to study genes required for rhizosphere colonization and plant infection will help to understand the competition problem observed in inoculant strains, by not only identifying new essential rhizosphere colonization genes, but also identifying mutations that provide a phenotypic growth advantage . Furthermore, the use of INSeq could be used to fully elucidate catabolic pathways, if saturating mutant pools were grown on minimal medium given a single carbon source, and contrasted with the results of similar experiments on rich media. In the near term, we will use the INSeq approach to increase our understanding of the gene networks involved in swarming physiology in RLV3841.

\section{Materials and Methods}

\section{Bacterial strains, growth conditions and plasmids}

The bacterial strains and plasmids used in this study are presented in Table 4. R. leguminosarum, S. meliloti, and A. tumefaciens were cultured at $30^{\circ} \mathrm{C}$ using tryptone-yeast extract medium (TY) [42]. E. coli strains were cultured on lysogeny broth $(\mathrm{LB})$ at $37^{\circ} \mathrm{C}$ [43]. When required, antibiotics were used at the following concentrations for Rhizobiaceae $500 \mu \mathrm{g} / \mathrm{mL}$ streptomycin (Str), $50 \mu \mathrm{g} / \mathrm{mL}$ rifampicin(Rif), $50 \mu \mathrm{g} / \mathrm{mL}$ neomycin (Neo); concentrations used for Escherichia coli were $100 \mu \mathrm{g} / \mathrm{mL}$ ampicillin (Amp), $25 \mu \mathrm{g} / \mathrm{mL}$ erythromycin, and $50 \mu \mathrm{g} / \mathrm{mL}$ kanamycin. The plasmid pSAM_Bt was obtained as a gift from Dr. Andrew L. Goodman. Plasmid pSC189 was obtained from addgene. org (plasmid\#: 32114) as kindly directed by Dr. Eric J. Rubin.

\section{Construction of pSAM_RI}

Plasmid DNA was isolated using GenElute ${ }^{\mathrm{Tm}}$ Plasmid Miniprep Kit (Sigma-Aldrich). The Neo/Kan ${ }^{\mathrm{R}}$ cassette within pSC189 [48] was PCR amplified using primers Tn189KmR_Fwd_XhoI and Tn189_Rev_XbaI (Additional file 1: Table S1) such that the $\mathrm{XhoI}$ and $\mathrm{XbaI}$ restriction enzyme sites were introduced on the 5' and 3' end, respectively. The $991 \mathrm{bp} \mathrm{Neo/Kan}{ }^{\mathrm{R}}$ PCR product was subsequently cloned into the $\mathrm{pGEM}^{\ominus}-\mathrm{T}$ Easy Vector System creating plasmid pGEM:: $189 \mathrm{Km}^{\mathrm{R}}$. The Neo/Kan ${ }^{\mathrm{R}}$ cassette in pGEM:: $189 \mathrm{Km}^{\mathrm{R}}$ was digested with $\mathrm{Xho} I$ and $\mathrm{XbaI}$ restriction enzymes creating a 979 bp fragment that was directionally cloned into the pSAM_Bt mariner transposon. The resulting plasmid pSAM_Km was maintained in the E. coli strain PIR1(Invitrogen), which allows for high copy number maintenance of R6Ky-oriR plasmids.

Cloning of the RLV3841 rpoD promoter region was carried out by PCR amplifying a 366 bp region upstream of the $r p o D$ gene start codon using primers Rlv_rpoD_Pro_Fwd and Rlv_rpoD_Pro_Rev (Additional file 1: Table S1). The $r p o D$ promoter PCR product had a 5 ' BamHI and 3' NdeI restriction enzyme site introduced, and was subsequently cloned into the $\mathrm{pGEM}^{\ominus} \mathrm{-T}$ Easy Vector System to create the plasmid pGEM::rpoD. The RLV3841 $r p o D$ promoter region was then excised from pGEM:: rpoD using NdeI and BamHI, and directionally cloned into pSAM_Km to create the vector pSAM_Rl (Figure 1). The new PSAM_Rl suicide vector carried an MmeIadapted mariner transposon harbouring a Neo ${ }^{\mathrm{R}} / \mathrm{Kan}^{\mathrm{R}}$ cassette, and had the himar1C9 transposase transcriptionally fused to a RLV3841 rpoD promoter. For use in transposon mutagenesis, the pSAM_Rl construct was electroporated into E. coli SM10גpir (obtained from Dr. Peter Howard, University of Saskatchewan).

\section{Testing PSAM_RI transposition frequency}

Transposition mutagenesis using pSAM_Rl was done in triplicate. Donor and recipient cells were grown in broth culture to an $\mathrm{OD}_{600}$ of approximately 0.8 and were pooled in a ratio of $1000 \mu \mathrm{L}$ recipient to $500 \mu \mathrm{L}$ of donor in a $1.5 \mathrm{~mL}$ microcentrifuge tube. The conjugation mixture was pelleted at $12,500 \mathrm{rpm}$ for $3 \mathrm{~min}$, washed once with $1000 \mu \mathrm{L} 1 \mathrm{X}$ PBS, and resuspended in approximately $100 \mu \mathrm{L} 1 \mathrm{X}$ PBS. The cell suspensions were then spotted onto pre-warmed TY agar plates and incubated at $30^{\circ} \mathrm{C}$ overnight. Conjugation spots were scraped and resuspended in $1000 \mu \mathrm{L}$ of $1 \mathrm{X}$ PBS. Enumeration of transposon mutants was done using TY agar supplemented with 
Table 4 Summary of bacterial strains and plasmids

\begin{tabular}{|c|c|c|}
\hline Strains & Characteristics & Reference \\
\hline R. leguminosarum bv. viciae 3841 & $\mathrm{Str}^{\mathrm{R}}$ wildtype & [44] \\
\hline S. meliloti RM1021 & SU47 str-21 Str ${ }^{\mathrm{R}}$ & [45] \\
\hline A. tumefaciensUBAPF2 & Plasmid-free derivative of $A$. tumefaciens strain $C 5$ Rif $^{R}$ & [46] \\
\hline E. coli SM10 גpir & thi-1 thr leu tonA lacY supE recA::RP4-2-Tc::Mu KanR $\lambda$ pir & [47] \\
\hline E. coli PIR1 & F- $\Delta$ lac169 rpoS(Am) robA1 creC510 hsdR514 endA- recA1 uidA(AMlul)::pir-116 & Invitrogen \\
\hline \multicolumn{3}{|l|}{ Plasmids } \\
\hline pSAM_Bt & $\begin{array}{l}\text { Amp }^{R} \text { Ery }^{R} \text {; RP4-oriT, oriR6K, mariner himar1C9 transposase with Bacteroides thetaiotamicron } \\
\text { rpoD promoter, Mmel-adapted marinerIR elements }\end{array}$ & [4] \\
\hline pSC189 & $\mathrm{Amp}^{\mathrm{R}} \mathrm{Kan}^{\mathrm{R}}$; RP4-oriT, oriR6K & [48] \\
\hline pGEM-T Easy & $\mathrm{Amp}^{\mathrm{R}}$; cloning vector & Invitrogen \\
\hline pGEM::189KmR & Amp $^{R}$ Kan $^{R}$; pGEM-T vector containing PCR amplified nptll gene from pSC189 & This Study \\
\hline pGEM::rpoD & Amp ${ }^{R} ;$ pGEM-T vector containing PCR amplified R. leguminosarum 3841 rpoD promoter region & This Study \\
\hline PSAM_Km & $\mathrm{Amp}^{\mathrm{R}} \mathrm{Kan}^{\mathrm{R}}$; Ery ${ }^{\mathrm{R}}$ in pSAM_Bt replaced with nptll from pSC189 & This Study \\
\hline pSAM_Rl & $\begin{array}{l}\text { Amp } p^{R} K{ }^{R} n^{R} ; \text { pSAM_Km with } B \text {. thetatiotamicron rpoD promoter replaced with } R \text {. leguminosarum } \\
3841 \text { rpoD promoter region }\end{array}$ & This Study \\
\hline
\end{tabular}

$50 \mu \mathrm{g} / \mathrm{mL}$ Neo and the appropriate Rhizobiaceae counter selectable antibiotic (Table 4). Enumeration of total Rhizobiaceae was done on TY agar with the species specific selectable antibiotic.

\section{Generating transposon mutant libraries for sequencing}

Six independent conjugations of pSAM_Rl into RLV3841 were conducted on TY agar as described above. After 24 hours incubation at $30^{\circ} \mathrm{C}$ each of 6 mating spot was scraped and resuspended in $1 \mathrm{~mL}$ of $1 \mathrm{X}$ PBS and then pooled together in a final volume of $6 \mathrm{~mL}$. For selection on TY agar, $1000 \mu \mathrm{L}$ of resuspended cells were plated across 2 separate $245 \times 245 \mathrm{~mm}^{2}$ (Corning) Neo + Str TY agar plates, in triplicate and incubated for $\sim 48$ hour at $30^{\circ} \mathrm{C}$.

Following incubation, a faint film like growth was scraped off each plate and resuspended in $1 \mathrm{~mL}$ of $1 \times \mathrm{PBS}$, vortexed for 1 minutes, and then pelleted at 15 , 000 RPM for 10 minutes. The supernatant of each resuspension was very viscous and still contained cells, it was equally aliquoted into $2 \times 1.5 \mathrm{~mL}$ microcentrifuge tubes. The original pellet, and two tubes of supernatant, were then brought up to a final volume of $1000 \mu \mathrm{L}$ with $1 \mathrm{M} \mathrm{NaCl}$, vortexed thoroughly, and incubated on ice for $1 \mathrm{~h}$ to disrupt the viscous exopolysaccharide diffuse capsule to better collect the cells. The $\mathrm{NaCl}$ cell suspensions were then pelleted at 15, 000 RPM for 10 minutes, and the pellets from each replicate were pooled independently and resuspended in $1000 \mu \mathrm{L}$ of TE buffer ( $\mathrm{pH}$ 8.0). The resulting 3 mutant pools were used for independent DNA isolation and downstream library preparation.

\section{Preparing sequencing libraries and DNA sequencing}

Transposon insertion tags (Tn-tags) consisted of $53 \mathrm{bp}$ of pSAM_Rl transposon sequence, including the $27 \mathrm{bp}$ inverse repeat sequence, and $15-16$ bp of adjacent genomic DNA. Library preparation was carried out

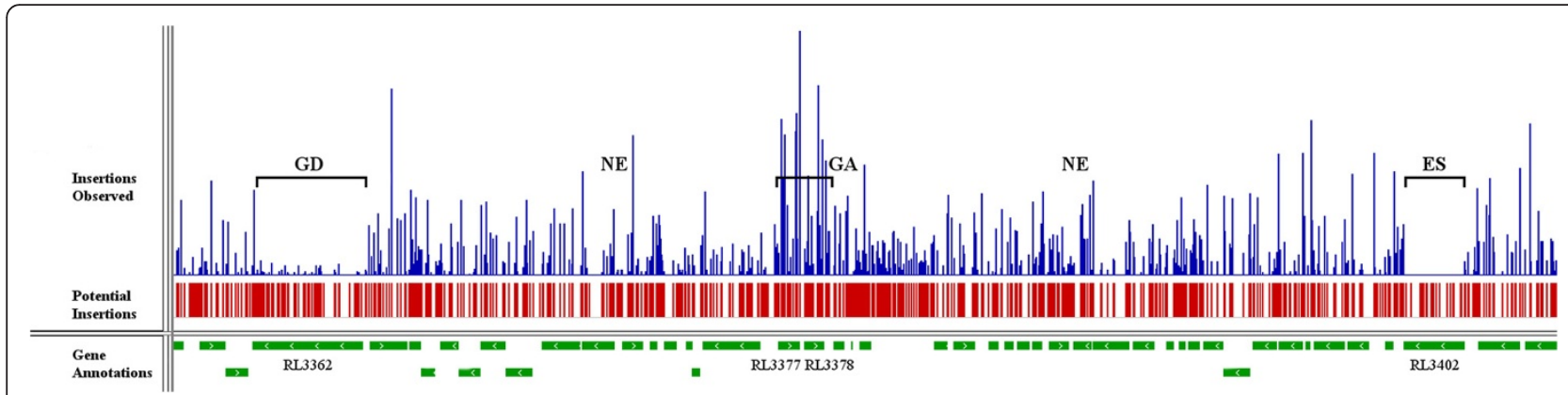

Figure $\mathbf{2}$ Transposon insertion density across a selected region of the RLV3841 genome. growth-defective, neutral, and growth-advantage regions of the RLV3841 genome. Analyzing the total number of insertions mapped to each potential mariner insertion site using the Tn-HMM python module [10] allows each gene to be placed in one of four phenotypic classes: ES = essential, GD = growth defect, NE = neutral, GA = growth advantage. In this experiment the phenotype refers to the ability to grow on TY agar. Data visualization was obtained using Integrative Genomics Viewer software [54]. Please refer to the Materials and Methods section for more detail on the process of assigning phenotypic classifications. 
independently for each of the 3 collected Tn-mutant pools. Tn-tags were prepared for DNA sequencing using a modified version of the INSeq method [49] to make the sequencing process amendable to the Ion Torrent PGM sequencing platform. Linear PCR products were amplified using the primer Ion Torrent BioSAM (Additional file 1: Table S1), with an annealing temperature of $58.6^{\circ} \mathrm{C}$ and $500 \mathrm{ng}$ of template DNA. Linear PCR products were purified using a QIAquick PCR Purification Kit (Qiagen) according to the manufacturer's recommended protocol. The biotinylated linear PCR products were then bound to Pierce Streptavidin Magnetic Beads (Thermo Scientific) and enzymatic steps during library preparation were performed as described [49] with the substitution of Klenow (New England Biolabs), Random Primer 6 (New England Biolabs) and T4 DNA Ligase (New England Biolabs). Additionally, a custom library adapter, INSEQ_Adpt, was used in the adapter ligation step. The final PCR amplification of sequencing template was accomplished using fusion primers designed in accordance with Ion Amplicon Library Preparation (Fusion Method, Pub\#: 4468326 Rev. C), using the PCR amplification conditions described in the INSeq methodology [49]. The forward fusion primers IT_A_FP_1, IT_A_FP_2, and $I T \_A \_F P \_3$ included IonXpress barcode sequences 1,2 , and 3 respectively, for downstream sequence separation. The reverse primer IT_trP1_FP was used in conjunction with a forward primer to introduce the trP1 sequencing adapter. The final sequencing template prepared from the Ion Amplicon library preparation was $187 \mathrm{bp}$ in length. Sequencing template was gel purified using the Invitrogen

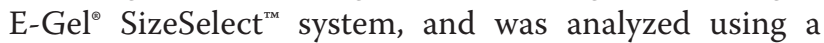
Bioanalyzer High Sensitivity DNA Chip (Agilent Technologies) prior to sequencing for quality and molarity. The three technical replicates had a final concentration of $1.37,1.82$, and $1.23 \mu \mathrm{g} / \mathrm{ul}$ of sequencing library after size selection, respectively.

DNA sequencing was performed on the Ion Torrent PGM using 200 bp sequencing chemistry and a 316v2 sequencing chip. The total raw sequencing output of the Ion Torrent was $1.25,1.73$, and 1.74 million reads for each of the 3 replicates. The raw sequencing reads were then pooled for downstream data extraction an analysis.

\section{Data extraction and transposon insertion analysis}

Quality trimming to Q20 and trimming of adapter sequences was performed using cutadapt [50] and the final 16-15 bp tn-tags were checked for a leading 'TA' motif using a custom python script. The resulting 3,192,486 transposon insertion tags were mapped to the $R$. leguminosarum bv. viciae 3841 reference genome (RefSeq:NC_008378.1 to NC_008384.1) [51] using the Bowtie short read aligner [52], allowing for no mismatches in the alignment, and only reporting insertion tags that mapped to a single unique location. Short read alignment resulted in 2,319,239 unique transposon insertion tags mapping to the RLV3841 reference genome, after 131,676 reads were ommitted due to multiple alignments, and 509,341 reads failed to align. The sam output file from the Bowtie alignment was converted into a bam format using Samtools [53], and was then converted to .bed format using bedtools. Transposon insertion reads were grouped by specific RLV3841 replicons for downstream analysis. The bed files of the aligned transposon insertion tags were converted to .wig format using a custom python module developed in house. The .wig formatted INSeq data sets generated for each of the 7 replicons in the RLV3841 genome were then analyzed independently using the Tn-HMM python module [10]. Briefly, the python module used a HMM as described in [10], in conjunction with the Viterbi algorithm to calculate the state of each 'TA' insertion site within the genome, independent of gene boundaries. Next, the computer module analyzed the state of successive 'TA' sites within gene boundaries to assign a state for the gene as a whole (See Additional file 2 for the RLV3841 chromosomal output). Four phenotypic classifications are possible: essential, growth defective, neutral, and growth advantage. Figure 2 provides a visual example of the four phenotypic classifications found within a selected region of the RLV3841 genome.

\section{Additional files}

\section{Additional file 1: Table S1. Primers and adapter sequences. \\ Additional file 2: Phenotypic classes (ES, GD, NE, GD) of RLV3841 chromosomal genes assessed during growth on TY medium, predicted using the Tn-HMM python module (Additional file 2.csv). The output of the Tn-HMM python module which provides the INSeq data using PSAM_RI to screen RLV3841 for growth on tryptone-yeast extract media.}

\section{Competing interests}

The authors declare that they have no competing interests.

\section{Authors' contributions}

BJP designed and performed all laboratory and computational work, as well as drafting the manuscript. CKY conceived the study, contributed to the experimental design, coordinated the project and finalized the manuscript. Both authors read and approved the final manuscript.

\section{Acknowledgements}

The authors gratefully acknowledge the support for this work from Natural Sciences and Engineering Research Council of Canada (NSERC) Discovery Grant to CKY. We would also like to thank Dr. Dinah Tambalo for her helpful discussions throughout the project.

Received: 13 July 2014 Accepted: 18 November 2014

Published online: 30 November 2014

\section{References}

1. Van Opijnen T, Camilli A: Transposon insertion sequencing: a new tool for systems-level analysis of microorganisms. Nat Rev Microbiol 2013, 11:1-8.

2. Barquist L, Boinett CJ, Cain AK: Approaches to querying bacterial genomes with transposon-insertion sequencing. RNA Biol 2013, 10:1161-1169.

3. Opijnen T, Bodi KL, Camilli A: Tn-seq: high-throughput parallel sequencing for fitness and genetic interaction studies in microorganisms. Nat Methods 2009, 6:767-775. 
4. Goodman AL, McNulty NP, Zhao Y, Leip D, Mitra RD, Lozupone CA, Knight $R$, Gordon JI: Identifying Genetic Determinants Needed to Establish a Human Gut Symbiont in Its Habitat. Cell Host Microbe 2009, 6:279-289.

5. Langridge GC, Phan M-D, Turner DJ, Perkins TT, Parts L, Haase J, Charles I, Maskell DJ, Peters SE, Dougan G, Wain J, Parkhill J, Turner AK: Simultaneous assay of every Salmonella Typhi gene using one million transposon mutants. Genome Res 2009, 19:2308-2316.

6. Gawronski JD, Wong SMS, Giannoukos G, Ward DV, Akerley BJ: Tracking insertion mutants within libraries by deep sequencing and a genomewide screen for Haemophilus genes required in the lung. Proc Natl Acad Sci 2009, 106:16422-16427.

7. Bishop AH, Rachwal PA, Vaid A: Identification of genes required by Bacillus thuringiensis for survival in soil by transposon-directed insertion site sequencing. Curr Microbiol 2014, 68:477-485.

8. Zhang YJ, loerger TR, Huttenhower C, Long JE, Sassetti CM, Sacchettini JC, Rubin EJ: Global assessment of genomic regions required for growth in Mycobacterium tuberculosis. PLoS Pathog 2012, 8:e1002946.

9. DeJesus MA, Zhang YJ, Sassetti CM, Rubin EJ, Sacchettini JC, loerger TR: Bayesian analysis of gene essentiality based on sequencing of transposon insertion libraries. Bioinformatics 2013, 29:695-703.

10. Dejesus MA, loerger TR: A hidden Markov model for identifying essential and growth-defect regions in bacterial genomes from transposon insertion sequencing data. BMC Bioinformatics 2013, 14:1-12.

11. Chao MC, Pritchard JR, Zhang YJ, Rubin EJ, Livny J, Davis BM, Waldor MK: High-resolution definition of the Vibrio cholerae essential gene set with hidden Markov model-based analyses of transposon-insertion sequencing data. Nucleic Acids Res 2013, 41:9033-9048.

12. Skurnik D, Roux D, Aschard H, Cattoir V, Yoder-Himes D, Lory S, Pier GB: A comprehensive analysis of in vitro and in vivo genetic fitness of Pseudomonas aeruginosa using high-throughput sequencing of transposon libraries. PLoS Pathog 2013, 9:e1003582.

13. Kamp HD, Patimalla-Dipali B, Lazinski DW, Wallace-Gadsden F, Camilli A: Gene fitness landscapes of Vibrio cholerae at important stages of its life cycle. PLoS Pathog 2013, 9:e1003800.

14. Wang N, Ozer EA, Mandel MJ, Hauser R: Genome-wide identification of Acinetobacter baumannii genes necessary for persistence in the lung. MBio 2014, 5:e01163.

15. Gallagher LA, Shendure J, Manoil C: Genome-scale identification of resistance functions in Pseudomonas aeruginosa using Tn-seq. MBio 2011, 2:e00315.

16. Brutinel ED, Gralnick JA: Anomalies of the anaerobic tricarboxylic acid cycle in Shewanella oneidensis revealed by Tn-seq. Mol Microbiol 2012, 86:273-83.

17. Griffin JE, Gawronski JD, Dejesus MA, loerger TR, Akerley BJ, Sassetti CM: Highresolution phenotypic profiling defines genes essential for mycobacterial growth and cholesterol catabolism. PLoS Pathog 2011, 7:e1002251.

18. Canals R, Xia X-Q, Fronick C, Clifton SW, Ahmer BMM, Andrews-Polymenis HL, Porwollik S, McClelland M: High-throughput comparison of gene fitness among related bacteria. BMC Genomics 2012, 13:212.

19. Klein BA, Tenorio EL, Lazinski DW, Camilli A, Duncan MJ, Hu LT: Identification of essential genes of the periodontal pathogen Porphyromonas gingivalis. BMC Genomics 2012, 13:578.

20. Phan M-D, Peters KM, Sarkar S, Lukowski SW, Allsopp LP, Moriel DG, Achard MES, Totsika M, Marshall VM, Upton M, Beatson SA, Schembri MA: The serum resistome of a globally disseminated multidrug resistant uropathogenic Escherichia coli clone. PLoS Genet 2013, 9:e1003834

21. Baugh L, Gallagher LA, Patrapuvich R, Clifton MC, Gardberg AS, Edwards TE, Armour B, Begley DW, Dieterich SH, Dranow DM, Abendroth J, Fairman JW, Fox D, Staker BL, Phan I, Gillespie A, Choi R, Nakazawa-Hewitt S, Nguyen MT, Napuli A, Barrett L, Buchko GW, Stacy R, Myler PJ, Stewart L, Manoil C, Voorhis W, Van Voorhis WC: Combining functional and structural genomics to sample the essential Burkholderia structome. PLOS One 2013, 8:e53851.

22. Fels SR, Zane GM, Blake SM, Wall JD: Rapid TnLE-seq for gene fitness evaluation in underdeveloped bacterial systems. Appl Environ Microbiol 2013, 79:7501.

23. Khatiwara A, Jiang T, Sung S-S, Dawoud T, Kim JN, Bhattacharya D, Kim H-B, Ricke SC, Kwon YM: Genome scanning for conditionally essential genes in Salmonella enterica serotype Typhimurium. Appl Environ Microbio/ 2012, 78:3098-107.

24. Christen B, Abeliuk E, Collier JM, Kalogeraki VS, Passarelli B, Coller JA, Fero MJ, McAdams HH, Shapiro L: The essential genome of a bacterium. Mol Syst Biol 2011, 7:528
25. Yanagi M, Yamasato K: Phylogenetic analysis of the family Rhizobiaceae and related bacteria by sequencing of $16 \mathrm{~S}$ rRNA gene using PCR and DNA sequencer. FEMS Microbiol Lett 1993, 107:115-120.

26. Wielbo J: Rhizobial communities in symbiosis with legumes: genetic diversity, competition and interactions with host plants. Cent Eur J Biol 2012, 7:363-372

27. Lupwayi NZ, Kennedy AC: Grain legumes in northern great plains. Agron J 2007, 99:1700

28. Pitzschke A: Agrobacterium infection and plant defense-transformation success hangs by a thread. Front Plant Sci 2013, 4(December):519.

29. Pulawska J: Crown gall of stone fruits and nuts, economic significance and diversity of its causal agents: tumorigenic Agrobacterium spp. J Plant Pathol 2010, 92:87-98

30. Beringer JE, Beynon JL, Buchanan-Wollaston AV, Johnston AWB: Transfer of the drug-resistance transposon Tn5 to Rhizobium. Nature 1978, 276:633-634.

31. Wilson KJ, Sessitsch A, Corbo JC, Giller KE, Akkermans AD, Jefferson RA: beta-Glucuronidase (GUS) transposons for ecological and genetic studies of rhizobia and other gram-negative bacteria. Microbiology 1995, 141(Pt 5):1691-705.

32. Reeve WG, Tiwari RP, Worsley PS, Dilworth MJ, Glenn AR, Howieson JG: Constructs for insertional mutagenesis, transcriptional signal localization and gene regulation studies in root nodule and other bacteria. Microbiology 1999, 145(Pt 6):1307-1316.

33. Ding H, Yip CB, Geddes BA, Oresnik IJ, Hynes MF: Glycerol utilization by Rhizobium leguminosarum requires an $A B C$ transporter and affects competition for nodulation. Microbiology 2012, 158(Pt 5):1369-78.

34. Oresnik IJ, Pacarynuk LA, O'Brien SAP, Yost CK, Hynes MF: Plasmid-encoded catabolic genes in Rhizobium leguminosarum bv. trifolii: evidence for a plant-inducible rhamnose locus linvolved in competition for nodulation Mol Plant Microbe Interact 1998, 11:1175-1185.

35. Geddes BA, Oresnik IJ: Inability to catabolize galactose leads to increased ability to compete for nodule occupancy in Sinorhizobium meliloti. J Bacteriol 2012, 194:5044-53

36. Vanderlinde EM, Harrison JJ, Muszyński A, Carlson RW, Turner RJ, Yost CK Identification of a novel $A B C$ transporter required for desiccation tolerance, and biofilm formation in Rhizobium leguminosarum bv. viciae 3841. FEMS Microbiol Ecol 2010, 71:327-40.

37. Vanderlinde EM, Muszynski A, Harrison JJ, Koval SF, Foreman DL, Ceri H, Kannenberg EL, Carlson RW, Yost CK: Rhizobium leguminosarum biovar viciae 3841 , deficient in 27-hydroxyoctacosanoate-modified lipopolysaccharide, is impaired in desiccation tolerance, biofilm formation and motility. Microbiology 2009, 155(Pt 9):3055-69.

38. Vanderlinde EM, Magnus SA, Tambalo DD, Koval SF, Yost CK: Mutation of a broadly conserved operon (RL3499-RL3502) from Rhizobium leguminosarum biovar viciae causes defects in cell morphology and envelope integrity. J Bacteriol 2011, 193:2684-94.

39. Plasterk RHA, Izsvák Z, Ivics Z: Resident aliens. Trends Genet 1999, 15:326-332.

40. Dong TG, Ho BT, Yoder-Himes DR: Identification of T6SS-dependent effector and immunity proteins by Tn-seq in Vibrio cholerae. Proc Natl Acad Sci 2013, 110:2623-2628.

41. Ding H, Hynes MF: Plasmid transfer systems in the rhizobia. Can $J$ Microbiol 2009, 55:917-927.

42. Beringer JE: R factor transfer in Rhizobium leguminosarum. J Gen Microbiol 1974, 84:188-98.

43. Sambrook J, Fritsch EF, Maniatis T: Molecular Cloning: A Laboratory Manual. 2nd edition. New York: Cold Spring Harbour; 1989.

44. Poole PS, Blyth A, Reid CJ, Walters K: myo-Inositol catabolism and catabolite regulation in Rhizobium leguminosarum bv. viciae. Microbiology 1994, 140:2787-2795.

45. Meade HM, Long SR, Ruvkun GB, Brown SE, Ausubel FM: Physical and genetic characterization of symbiotic and auxotrophic mutants of Rhizobium meliloti induced by transposon Tn5 mutagenesis. J Bacterio/ 1982, 149:114-22.

46. Hynes MF, Simon R, Pühler A: The development of plasmid-free strains of Agrobacterium tumefaciens by using incompatibility with a Rhizobium meliloti plasmid to eliminate pAtC58. Plasmid 1985, 13:99-105.

47. Miller VL, Mekalanos JJ: A novel suicide vector and its use in construction of insertion mutations: osmoregulation of outer membrane proteins and virulence determinants in Vibrio cholerae requires toxR. J Bacterio/ 1988, 170:2575-2583.

48. Chiang SL, Rubin EJ: Construction of a mariner-based transposon for epitope-tagging and genomic targeting. Gene 2002, 296:179-185. 
49. Goodman AL, Wu M, Gordon Jl: Identifying microbial fitness determinants by insertion sequencing using genome-wide transposon mutant libraries. Nat Protoc 2011, 6:1969-80.

50. Martin M: Cutadapt removes adapter sequences from high-throughput sequencing reads. EMBnet Journal 2011, 17:10

51. Young JPW, Crossman LC, Johnston AWB: The genome of Rhizobium leguminosarum has recognizable core and accessory components. Genome 2006, 7:R34

52. Langmead B, Trapnell C, Pop M, Salzberg SL: Ultrafast and memoryefficient alignment of short DNA sequences to the human genome. Genome Biol 2009, 10:R25.

53. Li H, Handsaker B, Wysoker A, Fennell T, Ruan J, Homer N, Marth G, Abecasis G, Durbin R: The Sequence Alignment/Map format and SAMtools. Bioinformatics 2009, 25:2078-9.

54. Robinson JT, Thorvaldsdóttir H, Winckler W, Guttman M, Lander ES, Getz G, Mesirov JP: Integrative genomics viewer. Nat Biotechnol 2011, 29:24-26.

doi:10.1186/s12866-014-0298-z

Cite this article as: Perry and Yost: Construction of a mariner-based transposon vector for use in insertion sequence mutagenesis in selected members of the Rhizobiaceae. BMC Microbiology 2014 14:298.

\section{Submit your next manuscript to BioMed Central and take full advantage of:}

- Convenient online submission

- Thorough peer review

- No space constraints or color figure charges

- Immediate publication on acceptance

- Inclusion in PubMed, CAS, Scopus and Google Scholar

- Research which is freely available for redistribution 